\section{Advantage of spontaneous breathing in patients with respiratory failure}

\author{
VIŠNJA MAJERIĆ KOGLER
}

\author{
VIŠNJA MAJERIĆ KOGLER ( $\square$ ) \\ Department for Anesthesiology, \\ Reanimatology and Intensive Care \\ University Hospital Zagreb \\ Kišpatičeva 12. \\ 10000 Zagreb, Croatia \\ Phone: 0038512388204 \\ Fax: 0038512421601 \\ E-mail: vkogler1944@yahoo.co.uk
}

\begin{abstract}
The fact that different modalities of mechanical ventilation are associated with a number of serious side effects and risks and can influence the clinical outcome of patients, the various modes of mechanical ventilation have, over the past ten years, been the subject of a wide variety of scientific studies. Many of these modalities are designed for partial ventilatory support, which might reflect the complexity of the issue of patient's ventilator interactions when spontaneous breathing activity is present, compared to controlled mechanical ventilation. Spontaneous breathing modes during mechanical ventilation may integrate intrinsic feedback mechanisms that should help prevent ventilator- induced lung injury and improve synchrony between the ventilator and the patient's demand. The improvements in pulmonary gas exchange, systemic blood flow, and oxygen supply to the tissue that have been observed when spontaneous breathing has been maintained during mechanical ventilation are reflected in the clinical improvement in the patient' s condition. It is the aim of this article to review the effects of preserved spontaneous breathing activity during mechanical ventilation in patients with acute respiratory failure.
\end{abstract}

Key words: mechanical ventilation, acute respiratory distress syndrome, ventilation mode, spontaneous breathing

\section{Introduction}

Mechanical ventilation is one of the life support procedures closely associated with the development of modern intensive care medicine.

The main goals of mechanical ventilation are to decrease the oxygen cost of breathing and to improve gas exchange while minimizing iatrogenic lung injury- socalled ventilator induce lung injury (VILI). (1) As mechanical ventilation becomes increasingly invasive, with high volumes and airway pressures being applied, it can contribute to the progression of existing lung damage. (2)

The fact that different modalities of mechanical ventilation are associated with a number of serious side effects and risks and can influence the clinical outcome of patients, the various modes of mechanical ventilation have, over the past ten years, been the subject of a wide variety of scientific studies. (3) Consequently, there is today a general consensus that ventilatory settings should be as non-invasive as possible. Patients with acute lung injury (ALI) or its most severe form, acute respiratory distress syndrome (ARDS), require mechanical ventilation. ARDS causes alveolar edema and collapse primarily in dependent lung regions adjacent to the diaphragm, resulting in intrapulmonary venous admixture of blood and severe arterial hypoxemia.

Traditionally, in the early phase of their disease, these patients are often ventilated with strategies that control the tidal volume or airway pressure. Mechanical ventilation with positive endexpiratory pressure (PEEP) and low tidal volume (VT) is commonly applied during ARDS to recruit collapsed alveoli for gas exchange without hyperinflation of the lungs. Deep sedation with neuromuscular blockade is generally used to adapt patients to controlled mechanical ventilation. Continuous positive pressure ventilation and PEEP help to improve arterial oxygenation but also affect the intrathoracic and extrathoracic vascular pressure gradients, such that return of the blood flow to the right ventricle is impaired and pulmonary vascular impedance is increased, resulting in enhanced right ventricular afterload. Both these mechanisms represent the major determinants for the depression of cardiac output, reduction of kidney and splanchnic perfusion, glomerular filtration and sodium excretion during mechanical ventilation. (4)

The actual process of weaning a patient from mechanical ventilation is carried out by allowing spontaneous breathing attempts or by gradually reducing mechanical assistance through the use of a ventilation mode, which supports spontaneous breathing. Although the ventilation modes designed to support spontaneous breathing were initially developed with the aim of facilitating and accelerating the weaning process, 
because of the clearly demonstrated benefit, they are increasingly being deployed as the primary mode of ventilation, even in patients with acute pulmonary dysfunction.

Spontaneous breathing modes may integrate intrinsic feedback mechanisms that should help prevent ventilatorinduced lung injury, improve synchrony between the ventilator and the patient's demand and improve cardiorespiratory stability.

It has been suggested that ventilatory modes in which patients breathe spontaneously early in the course of the ALI process might have advantages such as improved pulmonary ventilation perfusion (V/Q) match, excessive sedation, and prevention of ventilation- associated respiratory muscle dysfunction. $(5,6)$

\section{Benefits of maintained spontaneous breathing on arterial oxygenation and recruitment}

During controlled mechanical ventilation the diaphragm is relaxed and its displacement will mainly be in nondependent, anterior regions, because of the lower impedance of abdominal organs in the upper than in the lower abdominal regions. During spontaneous breathing, posterior muscle sections of the diaphragm move more than the anterior tendon plate. In parallel with the displacement of the diaphragm, ventilation seems to be distributed to upper, nondependent lung regions in mechanically ventilated subjects, in contrast to well known preferential distribution to dependent usually well- perfused lung regions during spontaneous breathing. Ventilation of a larger share of the lung along with an increase in blood flow to previously minimal or nonperfused areas may help convert shunt units to units with normal V/Q distribution.

Spontaneous breathing is possible in any phase of the mechanical ventilator cycle with airway pressure release ventilation (APRV), a technique that provides ventilatory support by way of timecycled switching between two different continuous positive airway pressure
(CPAP) levels. In patients with severe ALI, unsupported spontaneous breathing with APRV has been observed to improve arterial oxygenation over that in patients with controlled mechanical ventilation alone, or breath to- breath inspiratory assistance with pressure support ventilation. (7) Moreover, this clinical investigation and other experimental studies have shown a reduction in intrapulmonary shunting. $(8,9)$ Wrigge et al. recently demonstrated an increase in oxygenation and oxygen delivery as well as a substantial and progressive improvement of end-expiratory lung volume with spontaneous breathing, whereas oxygenation, oxygen delivery and end-expiratory lung volume remained low in the absence of spontaneous breathing in the porcine model of oleic acid-induced lung injury. Another major finding in this CT study was that spontaneous breathing was associated with considerably less non-aerated lung tissue and increased aeration. The negative correlation between end-expiratory lung volume and amount of nonaerated lung combined with the correlation between venous admixture suggest that recruitment of non- aerated lung tissue is the major factor for improvement of end-expiratory lung volume during APRV with spontaneous breathing and that the redistribution of gas to dependent, well perfused lung regions mainly explains improved oxygenation and reduction in intrapulmonary shunting during spontaneous breathing. $(10,11)$

When unhindered, spontaneous breathing of 10 to $30 \%$ of the total expired minute ventilation $\left(V_{E}\right)$ during APRV/ BIPAP (biphasic positive airway pressure) was compared with pressure support ventilation (PSV) in patients with ARDS, there was a decrease in the blood flow to shunt units $\left(V_{A} / Q<0.005\right)$ and an increase $(p<0.05)$ in the fraction of cardiac output to units with a normal ratio $\left(0.1<V_{A} / Q<10\right)$ without the appearance of poorly ventilated lung areas $\left(0,005<V_{A} / Q<0.1\right.$ This occurred when comparisons were made between equal pressure limits and equal minute volumes. As a result, arterial oxygenati- on increased during spontaneous breathing with APRV. These results confirm earlier investigations conducted in animals, which showed an improvement in intrapulmonary shunting and arterial oxygenation during spontaneous breathing with APRV/BIPAP. (12,13)

The reduction in intrapulmonary shunting and the increase in arterial oxygenation, in conjunction with increased pulmonary compliance, can be attributed to the recruitment of previously non-ventilated (atelectatic) lung areas. This assumption is corroborated by the results of studies on animals which showed a decrease in atelectasis demonstrated by computer tomography when one of the phrenic nerves was stimulated during anesthesia. (14) Furthermore, improved distribution of pulmonary perfusion during spontaneous breathing with APRV/BIPAP could contribute to a decrease in intrapulmonary shunting and an increase in arterial oxygenation. Spontaneous breathing during APRV/ BIPAP also decreases nonperfused but well ventilated dead-space areas and improves the $V_{A} / Q$ matching in other areas of the lung. (15)

As spontaneous breathing can obviously contribute to the recruitment of initially atelectatic lung areas, maintained spontaneous breathing with adequate mechanical support as per conventional ventilation strategies should, in terms of pulmonary gas exchange, be superior to controlled ventilation followed by weaning with respiratory support. Preliminary studies show that, in patients with multiple trauma who are at high risk of developing ARDS, maintained spontaneous breathing with APRV/ BIPAP results in lower venous admixture and better arterial oxygenation over an observation period of more than 10 days as compared to controlled ventilation with subsequent weaning. The incidence of atelactasis and pulmonary dysfunction is lower during maintained spontaneous breathing with APRV/ BIPAP. These results clearly show that, even in patients requiring mechanical ventilation, maintained and unhindered spontaneous breathing can counteract the progressive deterioration in pul- 
monary gas exchange as a result of alveolar collapse. (15)

\section{Benefits of maintained spontaneous breathing on cardiovascular side effects and organ perfusion}

Continuous positive pressure ventilation (CPPV) and PEEP help to improve arterial oxygenation but also affect the intrathoracic to extrathoracic vascular pressure gradients, such that return of blood flow to the right ventricle is impaired and pulmonary vascular impedance is increased, resulting in enhanced right ventricular afterload. The combination of both mechanisms is belived to represent the major determinants for the depression of cardiac output during mechanical ventilation.

The periodic reduction of intrathoracic pressure resulting from maintained spontaneous breathing during mechanical ventilatory support promotes the venous return to the heart and right-and left-ventricular filling, thereby increasing cardiac output and $\mathrm{O}_{2}$ transport capacity. Experimental and clinical studies show that in intermittent mandatory ventilation (IMV) and APRV/BIPAP, spontaneous breathing of 10 to $40 \%$ of the total expired minute ventilation at unchanged $V_{E}$ or airway pressure limits results in an increase in cardiac output. A simultaneous rise in right ventricular end-diastolic volume during spontaneous breathing in APRV/BIPAP is an indication of improved venous return to the heart. (15) Conversly, ventilatory support of each individual inspiration with pressure support ventilation and identical airway pressures produces no increase or very little increase in cardiac output. (15-17)

Kaplan et al. investigated whether APRV can safely enhance hemodynamics in patients with ALI-ARDS, relative to pressure control ventilation (PCV). During APRV, the cardiac index rose from 3,2+/0,4 for PCV to $4,6+/-0,3 / \mathrm{min} / \mathrm{m}^{2}$, whereas oxygen delivery increased from 997 +/-108 to $1409+/-146 \mathrm{ml} / \mathrm{min} / \mathrm{m}^{2}$, central venous pressure declined from $18+/-4$ $\mathrm{cmH}_{2} \mathrm{O}$ for PCV to $12+/-4 \mathrm{cmH}_{2} \mathrm{O}$ for APRV. Urine output increased by 0,83 for PCV to 0,96 for APRV. (18)
Theoretically, augmentation of the venous return to the heart and increased left-ventricular afterload as a result of reduced intrathoracic pressure should have a negative impact on cardiovascular function in patients with left-ventricular dysfunction. Indeed, switching abruptly from continuous mechanical ventilation (CMV) to PSV with a simultaneous reduction in airway pressure can lead to decompensation of existing cardiac insufficiency. However, providing that spontaneous breathing receives adequate support, and sufficient CPAP is applied, the maintenance of spontaneous breathing should not prove disadvantageous and, therefore, is not contraindicated even in patients with acute myocardial infarction and cardiac failure. (19) Hering et al. hypothesized that partial ventilatory support using APRV with spontaneous breathing provides better cardiopulmonary and renal function than full ventilatory support using APRV without spontaneous breathing. Effective renal blood flow and glomerular filtration rate were higher during APRV with spontaneous breathing (858 +/$388 \mathrm{ml} / \mathrm{min} / \mathrm{m}^{2}$ and $94+/-47 / \mathrm{min} / \mathrm{m}^{2}$ ) than during APRV without spontaneous breathing and the same minute ventilation $\left(714+/-236 / \mathrm{min} / \mathrm{m}^{2}\right.$ and $82+/-35 /$ $\left.\mathrm{min} / \mathrm{m}^{2} \mathrm{p}<0,05\right)$. Maintaining spontaneous breathing during ventilatory support may, therefore, be advantageous in preventing deteriation of renal function in patients with ARDS. (20)

\section{Benefits of maintained spontaneous breathing on analgosedation}

As well as ensuring sufficient pain relief and anxiolysis, the aim of analgosedation is to help the patient adapt to mechanical ventilation. Usually the level of analgosedation required during controlled ventilation is equivalent to a Ramsay score of 5 that is a deeply sedated patient who is unable to respond when spoken to and has no sensation of pain.

Conversely, when a ventilation mode is used which supports spontaneous breathing, a Ramsay score of 2 to 3 can be targeted, i.e. an awake, responsive and cooperative patient. (21) In a retrospective study of over 600 heart surgery patients, a reduction in consumption of analgesics and sedatives was observed when patients were allowed to breathe spontaneously from an early stage with APRV/BIPAP. Preliminary data show that maintaining spontaneous breathing with APRV/BIPAP in patients with multiple trauma over an observation period of more than 10 days leads to significantly lower consumption of analgesics and sedatives than when controlled ventilation is used for 72 hours followed by weaning. $(21,22)$ Obviously a large part of analgosedation is used exclusively to adapt patients to controlled mechanical ventilation. Both from a medical and from an economic point of view it would therefore appear sensible to provide mechanical support with spontaneous breathing

\section{Conclusion}

From the currently available data it can be concluded that maintained spontaneous breathing during mechanical ventilation should not be suppressed even in patients with severe pulmonary dysfunction. The improvements in pulmonary gas exchange, systemic blood flow, and oxygen supply to the tissue, which have been observed when spontaneous breathing has been maintained during mechanical ventilation, are reflected in the clinical improvement in the patient's condition. In comparison to an initial period of controlled ventilation for 72 hours following by weaning, maintained spontaneous breathing with APRV/BIPAP is associated with significantly fewer days on a ventilator, earlier extubation and shorter stays in the intensive care unit.

However, it should be pointed out that the positive effects of spontaneous breathing have only been documented for some of the clinically available ventilatory modes that support spontaneous breathing. If one limits oneself to ventilation modes whose positive effects have been scientifically documented, then partial ventilatory support can be used as a primary modality even in patients with severe pulmonary dysfun- 
ction. Whereas controlled mechanical ventilation followed by weaning with partial ventilatory support modes used to be regarded as the standard in ven- tilation therapy, this approach should be reconsidered in view of the available data. Today, standard practice should be to maintain spontaneous breathing from the very beginning of ventilatory support and to continuously adapt the ventilatory support to the patient's individual needs.

\section{REFERENCES}

1. Ranieri VM, Guinta F, Suter PM, Slutsky AS. Mechanical ventilation as a mediator of multisystem organ failure in acute respiratory distress syndrom. JAMA 2000;284:43-4.

2. The Acute Respiratory Distress Syndrom Network: Ventilation with lower tidal volumes as compared with traditional tidal volumes for acute lung injury and the acute respiratory distress syndrom. N Engl J Med 2000;342:1301-8.

3. Esteban A, Anzueto A, Frutos F, Alia I, Brochard L, Stewart TE, et al. Characteristics and outcome in adult patients receiving mechanical ventilation: a 28-day international study. JAMA 2002;287:345-55

4. Downs JB, Douglas ME, Sanfelippo PM, Stanford W, Hodges MR.Ventilatory pattern, intrapleural pressure, and cardiac output. Anesth Analg 1997;56:88-96

5. Hering R, Peters D, Zinserling J, Wrigge H, von Spielen T, Putensen C. Effects of spontaneous breathing during airway pressure release ventilation on renal perfusion and function in patients with acute lung injury. Intensive Care Med 2002;28:1426-33.

6. Putensen C, Zech S, Wrigge H, Zinserling J, Stuber F, von Spiegel T, et al. Long-term effects of spontaneous breathing during ventilatory support in patients with acute lung injury. Am J Respir Crit Care Med 2001;164:43-9.

7. Brandre L, Slutsky A. Assisted spontaneous breathing during early acute lung injury. Critical Care 2006;10:102-9.

8. Putensen C, Rasanen J, Lopez FA. Ventilation-perfusion distribution during mechanical ventilation with superimposed spontaneous breathing in canine lung injury. Am J Respir Crit Care Med 1994;150:101-8.

9. Putensen C, Mutz NJ, Putensen-himmer G, Zinserling J. Spontaneous breathing during ventilatory support improves ventilation-perfusion distributions in patients with acute respiratory distress syndrome. Am J Respir Crit Care Med 1999;159:1241-8.

10. Wrigge H, Zinserling J, Neumann P, Magnusson A, Putensen C, Hedenstietna G. Spontaneous breathing with airway pressure release ventilation favors ventilation in dependent lung regions and counters alveolar collapse in oleic acidf-induced lung injury: a randomized controlled computed tomography trial. Crit Care 2005;9:R780-R789.

11. Sydow M, Burchardi H, Ephraim E, Zielmann S, Crozier TA. Long-term effects of two different ventilatory modes on oxygenation in acute lung injury. Comparison of airway pressure release ventilation and volume-controlled inverse ratio ventilation. Am J Respir Crit Care Med 1994;149:1550-6.

12. Putensen C, Rasanen J, Lopez FA. Interfacing between spontaneous breathin and mechanical ventilation affects ventilation-perfusion distributions in experimental bronchoconstriction. Am J Respir Crit Care Med 1995;151:993-9.

13. Putensen C, Rasanen J, Lopez FA, Downs B. Effects of interfacing between spontaneous breathing and mechanical cycles on the ventilation-perfusion distribution in canine lung injury. Anesthesiology 1994;81:751-60.

14. Hedenstierna G, Tokics L, Lundquist H, Anderson T, Strandberg A, Brismar B. Phrenic Nerve stimulation during halothane anesthesia. Effects of atelectasis. Anesthesiology 1994;80:751-60.

15. Grinnan DC, Truwit JD. Clinical review: respiratory mechanics in spontaneous and assisted ventilation. Crit Care 2005;9:472-84.

16. Putensen C, Zech S, Zinserling J. Effects of early spontaneous breathing during airway pressure release ventilation on cardiopulmonary function. Am J Respir Crit Care Med 1998; 157:A45.

17. Kuhlen R, Rossaint R, Macintyre NR. The role of spontaneois breathing during mechanical ventilation. Respiratory Care 2002;47:296-307.

18. Kaplan LJ, Balley H, Formosa V. Airway pressure release ventilation increase cardiac performance in patients with acute lung injury adult respiratory distress syndrom. Crit Care 2001;5:221-6.

19. Myers TR, Macintyre NR. Respiratory controversies in the criticale care setting. Dose airway pressure release ventilation offer important new advantage in mechanical ventilatory support. Respiratory Care 2007;52:452-8.

20. Hering B, Peters D, Zinserling J, Wrigge H, von Spiegel T, Putensen C. Effects of spontaneous breathing during airway pressure release ventilation on renal perfusion and function in patients with acute lung injury. Intensive Care Med 2002;28:1426-33.

21. Bruchardi H. Aims of sedation analgesia. Minerva Anesthesiol 2004;70:137-43.

22. Grasso S, Fanelli V, Cafarelli A, Dalfino L, Ingenito G, Ancona G, et al. Patient ventilator interaction during PSV at different levels of sedation in ALI patients. Intensive Care Med 2004:30:A13. 